\title{
Plants: The Future Pharmaceutical Factory
}

\author{
Bipradut Sil*, Sumita Jha \\ Centre of Advanced Study in Botany, University of Calcutta, Kolkata, India. \\ Email: *bipradut@gmail.com
}

Received November $25^{\text {th }}$, 2013; revised December $26^{\text {th }}$, 2013; accepted January $21^{\text {st }}, 2014$

Copyright @ 2014 Bipradut Sil, Sumita Jha. This is an open access article distributed under the Creative Commons Attribution License, which permits unrestricted use, distribution, and reproduction in any medium, provided the original work is properly cited. In accordance of the Creative Commons Attribution License all Copyrights (c) 2014 are reserved for SCIRP and the owner of the intellectual property Bipradut Sil, Sumita Jha. All Copyright (C) 2014 are guarded by law and by SCIRP as a guardian.

\section{ABSTRACT}

The main objective of this study was to identify prospects, advantages and challenges of plant molecular farming. Plant-made pharmaceuticals (PMPs) and plant made vaccines (PMVs) were intended to be used for human consumption. A broad range of plants, including crops, vegetables and fruits have been investigated for their applicability in production of pharmaceuticals over the last two decades. At present, mainly maize, rice, tobacco and safflower are used in open field production. Contained bioreactor-type systems focus on moss, algae and plant organ culture. Expression systems include stable transformation of plant genomes-both nuclear and plastid, which are also used in the generation of genetically modified crops, plant-viruses and transient expressions. Each expression system (production platform) offers different advantages and disadvantages. Production platform choice is perceptive and depends on a broad range of criterion. This study is based on literature reviews, document analysis and internet database browsing.

\section{KEYWORDS}

\section{PMPs; Production Platform; Pharmaceuticals; Transformation; Gene Expression}

\section{Introduction}

The development of plant genetic transformation introduced the possibility of having plants to express "foreign" genes, and thereby accumulate non-native proteins in their cells and tissues. Thus the concept of plants producing pharmaceutical molecules was born, utilizing crop plants as production medium for useful and valuable proteins that originally derived from microbial or animal resources. Several advantages were claimed for such plant-based production, relative to bulk production of the natural source for the corresponding protein, including overall economy of production, low capital investment, easy economic scale-up, lower risk of contamination with human pathogens etc. Focusing plants as the production system became more compelling as the yield of protein per plant increased. Gene-expression technologies were developed specifically for achieving very high concentrations of "unfamiliar" proteins in plant tissues. These technologies, in turn, resulted in the materializa-

*Corresponding author. tion of several agro-biotech companies specializing in plant-made pharmaceutical productions. Varieties of proteins were expressed in a number of plant species, like industrial-enzymes, structural-polymers etc. Clinical trials in humans were conducted successfully with protein pharmaceuticals generated in plants [1]. The central point of this concept is its potential to provide enormous opportunities for both the agricultural sector and the biotechnology business sector at the same time.

Another variety of this idea is the concept of "edible vaccines” production in plants. In this strategy, the entire plant tissue or organ (such as a fruit) in which the protein pharmaceutical accumulates is the final delivery device for the protein product. Field-level production capabilities and post-harvest bio-processing capability were constructed. But, in spite of this advancement towards scaledup commercial applications, the plant-made pharmaceutical production sector continues to lack much-needed support. The fledgling molecular farming industry is ideally placed to address the resulting demand for protein pharmaceuticals, and consequently has re-characterized 
its technology platform as "plant-made pharmaceuticals". Applications to other proteins such as industrial enzymes, now referred to as "plant-made industrial products," are seen as a future priority.

New discoveries involving therapeutic proteins, such as monoclonal antibodies to treat various types of cancer, offer exciting and promising hope for a range of diseases. However, there are many challenges to produce these therapeutic proteins safely and economically. Current production methods include reproducing the target protein through plant cell cultures or bio-fermentation processes; expressing the protein in genetically altered animals, or growing the proteins in plants also modified using modern biotechnology.

\section{Prologue on Manufacturing Therapeutic Proteins}

The pharmaceutical industry is undergoing remarkable changes with the discovery and introduction of biotechnologically developed drugs. These are therapeutic proteins, such as monoclonal antibodies, blood proteins and enzymes that are produced by living organisms to fight disease. Unlike other medicines, they are not synthetically produced, but are usually produced through microbial fermentation or by mammalian cell culture. The process is more difficult, time-consuming. In 2010, approximately 30 plant-made pharmaceuticals (PMPs), including vaccines, antibodies, nutraceuticals, and therapeutic human proteins have entered the final stages of clinical trials prior to commercialization [1]. Four PMPs have already been launched on the market, in addition to three previously commercialized proteins derived from maize, bovine trypsin, avidin, and $\beta$-glucuronidase, all of which have been utilized in research laboratories worldwide since 1998 [2,3]. Since the development of recombinant DNA technology in the early 1970s, the commercial production of recombinant proteins has traditionally relied on microbial fermentation systems and transgenic mammalian cell culture. Although these systems are highly efficient in terms of productivity, but they present some disadvantages in terms of safety and production costs. These limitations have impelled the development of alternative production platforms in the past two decades based on the efficient scale-up, cost-effectiveness, high product quality, and low contamination risk presented by vegetable bioreactors, such as plant cell suspension, virus-infected plants and whole transgenic plants [4]. Plant bioreactors are potentially the most economical expression systems for the large-scale production of many molecules of pharmaceutical or industrial interest. Since 1986, when the first relevant plantmade biopharmaceutical-human growth hormoneswere synthesized by transgenic tobacco plants, more than
100 different proteins have successfully been expressed in different transgenic crops. These crops primarily include tobacco suspension cells, cereal and legume seeds, oilseeds, potato tubers, leafy crops, such as lettuce and spinach, and edible fruits, such as tomatoes and bananas [5]. Today, there are more than 400 new biotechnology medicines in the market. Cell cultures are grown in large stainless-steel fermentation under strictly maintained and regulated conditions. In some cases the proteins are secreted by the cells, in other cases the cells must be broken open so the protein can be extracted and purified.

\subsection{Conventional Process for Making Biotech Drugs}

Producing biotech drugs is a complicated and time-consuming process. Many years can be spent just to identify a therapeutic protein, determining its gene sequence and working out a process to make the molecule using sustainable biotechnology. Once the method is devised and scaled up, the biotech medicines can be produced in large scale. This is done by growing host cells that have been transformed to contain the gene of interest in carefully controlled conditions in large stainless-steel tanks. The cells are kept alive and stimulated to produce the target proteins through exact culture conditions that include a balance of temperature, oxygen, acidity and other variables. After careful culture, the proteins are isolated from the cultures, rigorously tested at every step of purification, and formulated into pharmaceutically-active products [1].

\subsection{Challenges of the Current Manufacturing Practices}

Traditional cell culture methods require large capital and labor investment. Cell culture facilities generally take three to five years to construct, cost \$250 million to \$450 million to construct and must be individually approved and certified by the Food and Drug Administration prior to full-scale operation. Also it is very expensive to maintain consistency in the manufacture and formulation of complex biotechnology medicines. Current manufacturing practices are facing a major global capacity shortage for the production of biotechnology medicines. Worldwide, there are fewer than two dozen facilities capable of large-scale biotech manufacturing; thus, the biotechnology industry faces a worldwide factory shortage. Furthermore, the capacity challenge is also expected to become more complex over the next decade. Today, there are only few protein-based medicines on the market and they already are outstripping the industry's production capacity. Additionally, with a growing aging population with chronic diseases, there will be a heightened demand for many protein-based medicines [1]. 


\subsection{Solution to Pharmaceutical Production Challenges}

Active ingredients in many protein medicines can be produced in crop plants more efficiently and economically than by using contemporary microbial or cell culture technologies. This new field of platforms may be able to successfully address the cost and potential supply challenges faced by current pharmaceutical production practices. Some analysts believe that this is especially true for monoclonal antibodies. Plant-made pharmaceuticals (PMPs) are the result of the application of biotechnology to create genetically enhanced plants to produce therapeutic proteins. In this process, plants themselves become "factories" that manufacture therapeutic proteins. These proteins are then extracted, refined and used as the active pharmaceutical ingredient in many medicines [1].

\section{Feasibility of the Process}

Since most proteins cannot be chemically synthesized, there are very few options for protein production: mammalian and microbial cell cultures and plants. Using plants to produce pharmaceutical proteins presents several clear advantages. First, there is significantly lower production costs associated with PMPs because the technology is developed in natural, renewable resources such as plants. Second, it is relatively easy to scale-up production to meet increased and varied demands. These two factors have the potential to provide greater and faster access to medicines. When producing these proteins in traditional cell cultures, there is an inherent risk of propagating human pathogens or other mammalian contaminants. But this technology offers a safe method to produce pharmaceutical proteins.

\subsection{Development}

Advances in biotechnology have made it possible to genetically enhance plants to produce therapeutic proteins essential for the production of a wide range of pharmaceuticals, such as monoclonal antibodies, enzymes, and blood proteins. These plants are grown under highly regulated conditions in confined growing environments and are strictly regulated by the US Department of Agriculture (USDA), its Animal and Plant Health Inspection Service (APHIS) and by the Food and Drug Administration (FDA). After the plants are harvested, they go through a series of processing steps that extract, separate, purify and package the therapeutic proteins [1]. The refined therapeutic proteins are ultimately used as the active pharmaceutical ingredients in many life-saving medicines.

\subsection{Expression Systems for Recombinant Protein Production}

Although most genes can be expressed in many different recombinant systems, it is crucial to determine the platform that offers the most advantageous conditions for the synthesis of a particular protein [6]. To achieve high expression levels of biologically functional protein at the lowest possible cost, a variety of conditions must be simultaneously satisfied. The most important of these conditions is the most suitable combination of host organism and target protein [7]. There are a wide variety of available expression systems for recombinant protein production, each with advantages and disadvantages. The nature and the particular properties of the different platforms can be explored in a complementary manner to increase the potential capacity to commercially produce a given protein [8]. The aspects of protein quality and functionality speed of production and product yield must be considered when choosing a suitable recombinant host for protein synthesis [9]. Prokaryotic systems based on microbe fermentation are the workhorses of the biopharmaceutical and enzyme industries. Generally, these systems allow high product yields at considerably low costs and are characterized by rapid growth and expression and ease of cultivation. Other advantages include recent progress in the understanding of the major genetic processes of diverse microorganisms and the complete characterization of many bacterial genomes, which permit the utilization of genetic tools to accurately modify microbial genomes, control promoter activity, modulate plasmid copy number, and manipulate many important metabolic processes and pathways [9]. These microbe properties for recombinant protein production have allowed the replacement of many natural sources of native proteins and enabled the production of engineered enzymes with modified properties. Furthermore, this platform has been applied to the modification of fibers and lipids and for more efficient ethanol and paper production and for several other applications [7]. Escherichia coli is one of the earliest and most widely explored hosts for the production of recombinant proteins. Transgenic $E$. coli cells were primarily utilized for production of human insulin in the late 1970s [10] and were approved by the USDA for commercial purposes under the name Humu$\operatorname{lin}^{\mathrm{TM}}$ to replace a less safe cattle-derived hormone. In addition to facilitating high levels of expression for many different proteins, the $E$. coli system presents some important drawbacks, especially if the protein of interest must be glycosylated and terminally processed. Prokaryotic cells lack the machinery to perform complex protein folding and assembly or post-translational modifications, such as $\mathrm{N}$ - or O-glycosylation, phosphorylation, lipid acylation, 
disulfide bond formation, and removal of the N-terminal methionine. These factors complicate the synthesis of complex eukaryotic proteins, which in many cases can also be toxic to the bacterial cell [11]. Other disadvantages of prokaryotic systems include toxicity that results from acetate accumulation in cultures with high bacterial cell density, as well as the production of proteins such as inclusion bodies, which are often non-functional, insoluble and require difficult refolding, and a high level of protein degradation mediated by endogenous proteases [9]. One strategy for minimizing these problems is to secrete the recombinant proteins into the peri-plasmic space or into the culture medium in which microbes are grown, an approach which has been commonly and successfully applied to systems based on $E$. coli cells and species of the genus Bacillus (B. megaterium, B. subtilis, $B$. licheniformis, and B. brevis), Ralstonia (R. eutropha), Pseudomonas (P. fluorescens), and Staphylococcus (S. carnosus) [9]. Yeasts have a long history in the fermentation industry and are frequently utilized to produce recombinant proteins that are not well-suited for synthesis by bacteria. Strains of the two most widely utilized yeast species in biotechnology, Saccharomyces cerevisiae and Pichiapastoris, are able to perform many post-translational modifications of abundantly expressed proteins at lower costs compared to insect and mammalian cells. Yeasts are fast and easy to grow, are genetically well characterized and can secrete nascent proteins into the extracellular medium after the proper removal of a specific signal peptide [12]. However, the glycosylation patterns of many mammalian recombinant proteins produced by yeast, primarily antibodies, are often distinct from their native forms. Thus, O-linked oligosaccharides added to the protein surfaces by yeast enzymes present only mannose and lack mammalian sialylated O-linked chains. In addition, the activity of yeast-derived recombinant proteins can be considerably altered by over-glycosylation of N-linked sites, which can also result in undesirable immunogenicity of yeast-derived proteins [13]. Foreign genes can also be stably integrated into the filamentous chromosomes of fungi as tandem repeats (as many as 100 copies have already been observed), and the transgenic fungal cells can secrete complete and fully active proteins with complex post-translational modifications and correct folding. In addition, some important proteins have already been expressed at high levels by species of the Aspergillus, Acremonium and Chrysosporium genera. The secreted yields of many recombinant proteins produced in fungal cells are lower compared to levels observed in other platforms, mostly because of transcription limitations, low stability of the corresponding mRNAs, and the abundance of naturally expressed fungal proteases [9]. More complex post-translational modifications can be obtained using insect cells that are genetically transformed with circular double-stranded DNA baculovirus and by mammalian cell suspensions. In particular, immortalized Chinese hamster ovary cells (CHOs) have been employed to produce typical mammalian molecules, such as monoclonal antibodies, erythropoietin and tissue plasminogen activator (tPA) [2,6]. Both systems contain the complex enzymatic mechanisms that are capable of phosphorylating tyrosine, threonine and serine hydroxyl groups, adding fatty acids to lipoproteins, folding proteins and establishing disulfide bonds. In addition, protein secretion and targeting do not present a problem when proper secretion signals are utilized. In most cases, both insect and mammalian cells provide high expression levels and productivity, usually reaching 20 - $60 \mathrm{pg}$ recombinant protein [9]. The major disadvantage of these recombinant platforms is the very high overall production cost. Another major concern is the risk of contamination of biopharmaceuticals derived from mammalian suspension cells with human pathogens, which contributes to considerably elevated production costs by requiring drug purification and sanitary approval processes [14]. Whole transgenic animals have emerged as promising systems for the production of high-quality proteins that are secreted into the milk, blood, urine, seminal plasma, egg white, and silk worm fibers. The secretion of biopharmaceuticals into the milk of transgenic lactating females is possible when the genes of interest are controlled by tissue-specific promoters that restrict protein expression to cells of the mammary gland [15]. Considerably high levels of diverse biopharmaceuticals have been obtained using different animals, such as transgenic mice, goats, cows, pigs, sheep, rabbits, and even fish [3]. A single transgenic cow secreting $5 \mathrm{~g}$ human growth hormone per liter of milk has the potential to annually produce $25 \mathrm{~kg}$ of the recombinant protein [9, 16], indicating that a herd of five animals could produce an amount sufficient to readily meet the annual demand of a large country, such as Brazil. Some drugs that are important for human health have already been produced using animals. The length of time required assessing production levels, assuming a successful gestation rate, and the long periods between lactation cycles constitute important challenges for heterologous protein production in transgenic animals [2]. Furthermore, the cost of maintenance of a single transgenic cow under Good Agricultural Practices is approximately US $\$ 10,000$ a year, resulting in prohibitively high overall costs for many of the biopharmaceuticals produced under these conditions [9]. In addition, most transgenic animals present a low capacity to scale up production and have intrinsic risks for transmission of pathogenic viruses, prions and oncogenes carried in milk, urine, semen, and blood. Over the past two decades, the alternative production of recombinant protein has turned to more effective and safer expression 
systems that are conducive to post-translational processing of these molecules at comparatively low production costs [14]. When dealing with large-scale production, transgenic plants are the most economical system for accurate expression of complex industrial proteins and recombinant drugs. Plant cells combine the potential for full-post-translational modification and correct folding with the simple growth requirements for water, minerals and light. Moreover, plants present a very high scalability when cultivated either in fields or greenhouses, and the technology for large-scale harvesting, processing and storage of whole plants and vegetable organs is already available for most cultivated crops, including soybeans [17]. The low risk of contamination with prions, viruses and other pathogens offers a major advantage of PMPs. Because plant pathogenic organisms cannot cause human disease, this opens the possibility of exploiting plants and edible fruits, such as bananas and papaya, as good candidates for the production of vaccines and orally administered antigens [2,12]. The major challenge of heterologous protein expression in plant systems is in overcoming the usually low yields of recombinant proteins that are synthesized by vegetable cells, normally ranging from $0.001 \%$ to $1 \%$ TSP (total soluble proteins), that results from low levels of transcript expression and recombinant protein instability [18]. Another drawback of plant systems lies in minor differences between the $\mathrm{N}$ glycolsylation patterns of plant versus mammalian-derived polypeptides. Such alterations can cause structural instability, inactivation, immunogenicity, and allergenicity of glycosylated plant-derived pharmaceuticals. Typical plant glycans, primarily $\beta-1,2$-xylose and $\alpha-1$, 3fucose, are frequently added to the $\mathrm{N}$ - or C-terminal of proteins that pass through the Golgi along the secretory pathway (Sethuraman and Stadheim, 2006). Localizing proteins to the ER lumen and inactivating the N glycosylation sites or knocking down Golgi glycosyltransferases can satisfactorily minimize these inconveniences [19].

\subsection{Popular Choices of Plants to Produce PMPs}

Plants such as alfalfa, barley, corn, duckweed, rice, safflower and tobacco have received APHIS regulatory permits for field trials [1]. These field trials are aimed at delivering the next generation of essential proteins for life-saving medicines. Plants that also can be used for food crops are a natural choice for PMP production because researchers have extensive agricultural knowledge and familiarity of these plants, as well as experience with their growth. Scientists have a vast understanding of genetics, agronomics and the environmental impact these plants have, as well as their composition. This information is crucial in developing methods for confining and managing these plants [17].

\section{Plant Pharmaceutical Production by Synthetic Biology Approach}

For many therapeutic and industrial proteins, glycosylation is an essential post-translational modification required for proper biological function. The correct addition of superficial glycans by the enzymatic machinery of the host organism directly influences protein stability, solubility, folding and, consequently, in vivo activity [17]. While N-glycan addition in the ER is conserved among eukaryotes, glycosylation in the Golgi is kingdom-specific and results in superficial linkage of a variety of different carbohydrate structures to various glycoproteins. Although plant expression systems provide the correct glycosylation of many proteins, especially those that naturally occur in bacteria, yeast and insects, the biosynthesis of mammalian proteins in vegetable bioreactors frequently requires more attention when complex glycosylation is required [20]. Plants and mammals glycosylate proteins on the same common Asn residues and the high-mannose type N-glycans are structurally identical in both systems. However, the complex N-glycans added by the plant and mammal machineries are quite different from one another. Usually, the mammalian glycans $\alpha-1$, 6 -fucose and $\beta$-1, 4-N-acetylglucosamine are respectively substituted by the plant glycans $\alpha-1,3$-fucose and a bisecting $\beta-1$, 2-xylose during the exchange of proximal $\mathrm{N}$-acetylglucosamine and the $\beta$-mannose in the Golgi of plant cells [21]. Another important difference in the processing of the terminal $\mathrm{N}$-acetylglucosamines in plants is the attachment of two types of glycans: the $\beta-1$, 3-galactose and the $\alpha-1$, 4-fucose, which lack the mammalian $\beta$-1, 4-galactose and sialic acid, molecules that are not detected in proteins derived from plants. This can cause the rapid elimination of PMPs from the blood and immunogenicity in humans and can have major negative impacts on the specificity of most IgE- and IgG-antibodies produced in plants [13]. Several strategies to humanize the structure of plant N-linked glycans can be utilized to allow the production of recombinant therapeutic glycoproteins in plants. Strategies are primarily focused on the control of plant-specific N-glycan maturation and on preventing the addition of immunogenic glycoepitopes onto PMPs. One of the most promising approaches is to directly mutate the Asn or Ser or Thr residues that are the major sites of superficial N-glycolsylation. This approach is well-suited for the production of antigens and simple PMPs that do not have in vivo activity or high longevity, but is not recommended for more complex glycoproteins [13]. In addition, the inhibition of plant-specific Golgi glycosyltransferases can successfully prevent the addition of glyco-epitopes to recombinant PMPs. This can be achieved by the inactivation of the $\alpha-1$, 3-fucosyltransferase and $\beta-1,2$-xylosyltransferase genes using insertional mutations or by 
exploiting the RNA interference method, a highly effective approach for homogeneous glycosylation of humanized antibodies in plants [22]. The combined knockout of four different glycosyl transferases in transgenic soybean plants developed by Embrapa is currently been evaluated as an efficient tool for the humanization of glycan-epitopes in recombinant proteins accumulated in seeds [17]. Another important point is to exploit the structural similarity of the high-mannose $\mathrm{N}$-glycans added in ER lumen of eukaryotic cells. The addition of $\mathrm{N}$ - or C-terminal signal peptides (such as the KDEL) to retain proteins in the ER, avoiding processing in the Golgi, can not only improve the stability but can also influence the addition of exclusively non-immunogenic high-mannose type N-glycans to the protein surface [22]. The remodeling of biosynthetic pathways to obtain specific metabolic products, normally by adding extra-enzymatic reaction stages, is another attractive strategy to humanize plant $\mathrm{N}$-glycans. The most obvious way to do this is to express mammalian glycosyltransferases in plants, a process that triggers competition between the newly synthesized enzymes and the endogenous machinery for $\mathrm{N}$-glycan processing in the Golgi apparatus. For example, the addition of galactose residues onto the terminal N-acetylglucosamine residues of plant $\mathrm{N}$-glycans by recombinant human $\beta$-1, 4-galactosyltransferase has consistently resulted in a glycan pattern in plant cells similar to that in mammalian cells [19]. In addition, the production of sialylated $\mathrm{N}$-glycans is feasible in plants because many Golgi complex biosynthetic pathways have literally been re-designed by expression of mammalian $\alpha$-2, 6-sialyltransferase, the human CMP-N-acetylneuraminic acid synthetase, the CMP-sialic acid transporter and two N-acetylneuraminic acid synthesizing enzymes [13]. The modification of metabolic pathways through the use of recombinant DNA technology can also be applied for phenotypic improvements of important crops utilized as renewable feedstocks for the production of biofuels. To fulfill the exacting demands of the fuel industry, a highquality biodiesel must have a high oleic acid concentration and low saturated fatty acid content, providing more stability at high frying temperatures during processing, and a longer shelf life than the conventional commodity oil [17]. Although significant efforts have led to some success in the introgression of these desirable characteristics in elite varieties of maize, canola and soybeans, by exploiting the endogenous germplasm variation of these plants, conventional breeding for oil content modification has some drawbacks. This is especially in terms of environmental influences over the new traits, as the link between the novel fatty acid profile to a reduced oil yield and that the oleic acid concentration in the seeds tends to be a multi-loci inheritance and is thus difficult to manipulate [23].

\section{Disease Treatment by Using PMPs}

Plants improved through the use of biotechnology can produce the essential building blocks (therapeutic proteins) for innovative treatments for diseases such as Alzheimer's disease, cancer, chronic obstructive pulmonary disease (COPD), Crohn's disease, cystic fibrosis, diabetes, geriatric and child diarrhea, heart disease, Hepatitis C, HIV, iron deficiency, kidney disease, multiple sclerosis, obesity, rheumatoid arthritis, spinal cord injuries etc. [1].

\section{Plant Made Edible Vaccines}

Edible vaccines are developed through the use of biotechnology, but are not necessarily the same as PMPs. PMPs produce proteins that will be used in the manufacture of medicines. Therapeutic proteins produced in PMP field trials are usually not intended to be administered via food. They are administered orally, entirely or topically. Edible vaccines, on the other hand, are vaccines produced in food crops, which can be orally administered in proper doses. While different from PMPs, they are similar in that neither is to be consumed as part of the food supply, and edible vaccines will be administered only under the supervision of physicians or licensed healthcare workers [1].

\section{Safety Measures of PMPs from the Other Agro-Products}

Protein-producing plants simply are "production factories" and are one step in the pharmaceutical manufacturing process and are handled completely outside the commodity food and feed stream. PMPs leverage much of the agricultural biotechnology applications and knowledge used in genetically enhanced food and feed, but for an entirely different purpose and end-use. Agricultural biotechnology uses advanced plant breeding techniques and tools of biotechnology to introduce beneficial traits to crops grown for food, feed and fiber. Many foods and feeds improved through the use of biotechnology possess beneficial characteristics, such as higher yield, better nutrition and resistance to disease. Examples of agricultural biotechnology for consumption purposes include "golden rice," which produces pro-Vitamin A, and grains with improved oil content. The science used to produce proteins in plants represents the new era of biopharmaceutical manufacturing and differs from traditional commodity agriculture on many fronts. PMP research does not represent a new wave of value-added commodity agriculture. The production and handling of pharmaceutical-containing plants is strictly regulated under rigorous federal guidelines for safety of humans and the environment. Pharmaceutical protein-containing plants are grown and processed separately from food and feed crops, a system known as "confinement" or in a com- 
pletely closed loop system. In addition, only a few select growers are identified, trained and supervised to grow these prescription plants. The seeds are only available from the manufacturer and cannot be purchased off the shelf at a local seed store. The production and handling of pharmaceutical-containing plants is strictly regulated under rigorous federal guidelines for the safety of humans and the environment. Pharmaceutical-containing plants are grown and processed separately from food and feed crops-a system know as confinement, or in a completely closed loop system. Confinement includes procedures to prevent commingling with food or feed crops, the environment, humans and non-target organisms. Confinement procedures are based on scientific risk assessments that evaluate the potential for and impact of exposure. The PMP industry has named its risk assessment Containment Analysis and Critical Control Point (CACCP). These risk assessments developed for plantmade pharmaceuticals take into account the crop, the specific protein, the spatial setting or location of the intended production area and agronomic and crop handling practices. Confinement measures include both spatial isolation and temporal separation. Spatial confinement defines the distance between plots for plants producing pharmaceutical proteins and conventional crops used for food and feed. Temporal boundaries define the time separating the flowering and pollination between plants producing pharmaceutical proteins and nearby conventional crops of the same or related species. Additionally, farm equipment that is used for these types of plants cannot be used for any food or feed crops. The annual training of contract growers and all other individuals involved with the development and production of PMPs is an Animal and Plant Health Inspection Service (APHIS) regulatory requirement. An APHIS-approved training program ensures personnel are prepared to successfully implement and comply with all permit conditions. Federal regulations are designed to prevent protein-producing plants from crossing paths with crops used for food and feed production, making it highly unlikely for commingling to occur [1].

\section{Advantages}

Traditional methods of producing pharmaceutical materials using microbial fermentation or animal cells are limited by the time and money necessary to build the required manufacturing facilities, and production is limited to manufacturing capacity. It can take a total of five to seven years from laying the first bricks of a traditional biotech facility to extracting pharmaceutical proteins from cultivated cells. In many cases, it will cost significantly less to grow plants with the ability to massproduce pharmaceutical proteins because plant-based techniques don't require the same costly capital invest- ments. Plant-made pharmaceutical production can also be more easily expanded to provide larger quantities if demand for the drug increases. Additionally, because plants are renewable in nature and can produce pharmaceutical proteins within a single growing season, production can be quickly scaled up to meet patient needs. Production and cost advantages of plant-made pharmaceuticals can allow more capital to be invested in research and development of new therapeutics, giving patients access to new drugs faster. In addition, expanded manufacturing capacity of high-quality proteins will spur development of more medicines by removing a key hurdle to mass production. Furthermore, cultivating cells for protein production with traditional fermentation methods is complicated with many risks involved. Cells must be carefully monitored to ensure proper development and survival, and the alternate source, mammalian cells, are at risk for contamination and the potential transmission of animal-based pathogens and viruses [8]. Under special cultivation conditions in simple synthetic liquid media using conventional bioreactors, undifferentiated plant cells can be separated and propagated as specialized factories for the production of secondary metabolites and, more recently, human-like therapeutic proteins [24]. The production of biopharmaceuticals in plant cell suspensions is cheaper, safer and faster than production in other eukaryotic suspension cell systems. Most stable productive lines can be generated within only a few months because they originate from cells that can double their concentration in just a single day and do not need to be regenerated and characterized as whole transgenic plants [17]. The system permits a high degree of containment and facilitates simple downstream processing with minimal heterogeneity of N-glycosylation due to the uniformity of the cells utilized in single cultures, which are usually composed of cells of Arabidopsis thaliana, tobacco, carrot, and rice [3]. Furthermore, no systemic post-transcriptional gene silencing is required due to the absence of plasmodesmata and the vascular system in undifferentiated culture cells [24]. Transient expression systems are very useful for research and are now being routinely used for the production of many valuable proteins at very low cost. These systems allow high throughput production and straightforward manipulation, permitting the rapid validation of expression constructs and the production of large amounts of recombinant protein within a few weeks. As a direct consequence, the protein yields from transient expression in plants are normally 10 -fold higher than yields observed in other recombinant plant systems [25]. Thus, some molecules that have already been successfully obtained from infected tobacco leaves are human Growth Hormone (hGH), seven single-chain antibodies, alpha interferon, and some bacterial antigens etc. [25]. Many biotech corporations are cur- 
rently evaluating the commercial production of an idiotype vaccine against B-cell non-Hodgkin's lymphoma using this technology [18]. However, transiently expressed proteins must be processed immediately after harvest to prevent tissue degradation and protein turnover. The induction of expression is also technically demanding because Agrobacterium inoculation must be performed mechanically or sprayed, and the effectiveness of viral strains to promote proper infection must be established $[2,3,26]$. These limitations can be minimized when the host system provides a steady expression level of the transgenes mediated by stable nuclear or plastid transformation [25]. A desirable characteristic is that chloroplasts are devoid of pollen and are inheritable through the maternal tissue, thus providing natural containment of transgene flow by out-crossing [3]. Expression systems that utilize plastid transformation seem to be welladapted for the accumulation of simple proteins that do not require complex post-translational modifications, especially glycosylation, as the chloroplast processing machinery closely resembles that of bacteria. This can be a crucial limitation when the protein of interest must be glycosylated in order to be biologically active. Moreover, proteins that are overexpressed in chloroplasts may form inclusion bodies and must be refolded after solubilization, which can contribute to an increase in overall production costs [18]. Although plastid transformation offers many practical advantages for molecular farming, it is limited to a few species, such as eggplant, tomato, lettuce, and soybean. Tobacco is the crop that is most routinely utilized for the expression of foreign genes in chloroplasts, but it is inedible and rich in toxic alkaloids, which can restrict its utility as a bioreactor for biopharmaceuticals [3]. Stable nuclear transformation is the most common approach utilized in plant molecular farming and is applicable to a wide range of vegetable species, from cereals to grain legumes, including edible fruits, leafy crops, and oilseeds. This method permits the stable integration of recombinant genes into the host nuclear genome and allows for the continual production of recombinant protein as a conferrable, inheritable trait [17]. Apart from acquiring in vitro chimeric plants, stably transformed plants obtained in the laboratory can be repeatedly cultivated in fields or greenhouses to generate transgenic expression lines and can be utilized to transfer a desirable trait to conventional plants through controlled breeding [27]. Because the most important factor related to the development of plant biotechnology products is ensuring competitive production costs and large-scale manufacturing, boosting heterologous expression in transgenic plants is the best way to achieve the production yields required for commercialization [7,26,27].

\section{Conclusion}

No single platform technology has yet risen to dominate the field of plant made pharmaceuticals in the way Escherichia coli dominates the bacterial field and CHO cells dominate among the mammalian cell lines. Indeed, industry is faced with a puzzling array of choices, starting with whole transgenic plants against transient expression systems, viral vectors, contained plant cells and microbial plants, and then if transgenic plants are chosen as a range of different species including leafy crops, fruits, vegetables, oil crops, legumes, cereals, model species, mosses and aquatic plants. Among these choices, cereal crops provide a unique combination of advantages -high biomass yield, scalability, unmatched protein stability, no interference with vegetative growth and the convenience of large scale processing infrastructure even in developing countries, where plant-made pharmaceuticals are likely to have the greatest positive impact.

\section{Acknowledgements}

Bipradut Sil acknowledges the Council of Scientific and Industrial Research (CSIR) for the award of a senior research fellowship.

\section{REFERENCES}

[1] Biotechnology Industry Organization (BIO), 2011. http://www.bio.org/search/node/plantmadepharmaceutical $\underline{\mathrm{s}}$

[2] J. K. Ma, P. M. Drake and P. Christou, "The Production of Recombinant Pharmaceutical Proteins in Plants," $\mathrm{Na}$ ture Reviews Genetics, Vol. 4, No. 10, 2003, pp. 794-805. http://dx.doi.org/10.1038/nrg1177

[3] O. O. Obembe, J. O. Popoola, S. Leelavathi and S. V. Reddy, "Advances in Plant Molecular Farming," Biotechnology Advances, Vol. 29, No. 2, 2011, pp. 210-222. http://dx.doi.org/10.1016/j.biotechadv.2010.11.004

[4] R. Fischer, E. Stoger, S. Schillberg and P. Christou, "PlantBased Production of Biopharmaceuticals,” Current Opinion in Plant Biology, Vol. 7, No. 2, 2004, pp. 152-158. http://dx.doi.org/10.1016/j.pbi.2004.01.007

[5] A. Spök, R. M. Twyman, R. Fischer and J. K. Ma, “Evolution of a Regulatory Framework for Pharmaceuticals Derived from Genetically Modified Plants," Trends in Biotechnology, Vol. 26, No. 9, 2008, pp. 506-517. http://dx.doi.org/10.1016/j.tibtech.2008.05.007

[6] H. Daniell, S. J. Streatfield and K. Wycoff, "Medical Molecular Farming: Production of Antibodies, Biopharmaceuticals and Edible Vaccines in Plants," Trends in Plant Science, Vol. 6, No. 5, 2001, pp. 219-226. http://dx.doi.org/10.1016/S1360-1385(01)01922-7

[7] S. J. Streatfield, "Approaches to Achieve High-Level Heterologous Protein Production in Plants,” Plant Biotechnology Journal, Vol. 5, No. 1, 2007, pp. 2-15. http://dx.doi.org/10.1111/j.1467-7652.2006.00216.x

[8] T. Kawakatsu and F. Takaiwa, "Cereal Seed Storage Protein Synthesis: Fundamental Processes for Recombinant Protein Production in Cereal Grains,” Plant Biotechnolo- 
gy Journal, Vol. 8, No. 9, 2010, pp. 939-953. http://dx.doi.org/10.1111/j.1467-7652.2010.00559.x

[9] A. L. Demain and P. Vaishnav, "Production of Recombinant Proteins by Microbes and Higher Organisms," Biotechnology Advances, Vol. 27, No. 3, 2009, pp. 297-306. http://dx.doi.org/10.1016/j.biotechadv.2009.01.008

[10] D. V. Goeddel, D. G. Kleid, F. Bolivar and H. L. Heyneker, "Expression in Escherichia coli of Chemically Synthesized Genes for Human Insulin,” Proceedings of the National Academy of Sciences of the United States of America, Vol. 76, No. 1, 1979, pp. 106-110. http://dx.doi.org/10.1073/pnas.76.1.106

[11] S. R. Karg and P. T. Kallio, "The Production of Biopharmaceuticals in Plant Systems," Biotechnology Advances, Vol. 27, No. 6, 2009, pp. 879-894. http://dx.doi.org/10.1016/j.biotechadv.2009.07.002

[12] J. Boothe, C. Nykiforuk, Y. Shen and S. Zaplachinski, "Seed-Based Expression Systems for Plant Molecular Farming,” Plant Biotechnology Journal, Vol. 8, No. 5, 2010, pp. 588-606. http://dx.doi.org/10.1111/j.1467-7652.2010.00511.x

[13] V. Gomord, A. C. Fitchette, L. Menu-Bouaouiche and C. Saint-Jore-Dupas, "Plant-Specific Glycosylation Patterns in the Context of Therapeutic Protein Production," Plant Biotechnology Journal, Vol. 8, No. 5, 2010, pp. 564-587. http://dx.doi.org/10.1111/j.1467-7652.2009.00497.x

[14] L. M. Houdebine, "Production of Pharmaceutical Proteins by Transgenic Animals," Comparative Immunology, Microbiology and Infectious Diseases, Vol. 32, No. 2, 2009, pp. 107-121. http://dx.doi.org/10.1016/j.cimid.2007.11.005

[15] J. W. Larrick and D. W. Thomas, "Producing Proteins in Transgenic Plants and Animals," Current Opinion in Biotechnology, Vol. 12, No. 4, 2001, pp. 411-418. http://dx.doi.org/10.1016/S0958-1669(00)00236-6

[16] D. Salamone, L. Baranao, C. Santos and L. Bussmann "High Level Expression of Bioactive Recombinant Human Growth Hormone in the Milk of a Cloned Transgenic Cow,” Journal of Biotechnology, Vol. 124, No. 2, 2006, pp. 469-472. http://dx.doi.org/10.1016/j.jbiotec.2006.01.005

[17] G. R. Vianna, N. B. Cunha, A. M. Murad and E. L. Rech "Soybeans as Bioreactors for Biopharmaceuticals and Industrial Proteins,” Genetics and Molecular Research, Vol. 10, No. 3, 2011, pp. 1733-1752. http://dx.doi.org/10.4238/vol10-3gmr1476

[18] R. Tremblay, D. Wang, A. M. Jevnikar and S. Ma, “To- bacco, a Highly Efficient Green Bioreactor for Production of Therapeutic Proteins," Biotechnology Advances, Vol. 28, No. 2, 2010, pp. 214-221. http://dx.doi.org/10.1016/j.biotechadv.2009.11.008

[19] V. Gomord, C. Sourrouille, A. C. Fitchette and M. Bardor, "Production and Glycosylation of Plant-Made Pharmaceuticals: The Antibodies as a Challenge," Plant Biotechnology Journal, Vol. 2, No. 2, 2004, pp. 83-100. http://dx.doi.org/10.1111/j.1467-7652.2004.00062.x

[20] D. Lienard, C. Sourrouille, V. Gomord and L. Faye, "Pharming and Transgenic Plants,” Biotechnology Annual Review, Vol. 13, 2007, pp. 115-147. http://dx.doi.org/10.1016/S1387-2656(07)13006-4

[21] V. Gomord and L. Faye, "Posttranslational Modification of Therapeutic Proteins in Plants," Current Opinion in Plant Biology, Vol. 7, No. 2, 2004, pp. 171-181. http://dx.doi.org/10.1016/j.pbi.2004.01.015

[22] N. Sethuraman and T. A. Stadheim, "Challenges in Therapeutic Glycoprotein Production," Current Opinion in Biotechnology, Vol. 17, No. 4, 2006, pp. 341-346. http://dx.doi.org/10.1016/j.copbio.2006.06.010

[23] G. Graef, B. J. LaVallee, P. Tenopir and M. Tat, “A High-Oleic-Acid and Low-Palmitic-Acid Soybean: Agronomic Performance and Evaluation as a Feedstock for Biodiesel,” Plant Biotechnology Journal, Vol. 7, No. 5, 2009, pp. 411-421.

http://dx.doi.org/10.1111/j.1467-7652.2009.00408.x

[24] J. Xu, X. Ge and M. C. Dolan, "Towards High-Yield Production of Pharmaceutical Proteins with Plant Cell Suspension Cultures,” Biotechnology Advances, Vol. 29, No. 3, 2011, pp. 278-299. http://dx.doi.org/10.1016/j.biotechadv.2011.01.002

[25] Y. Gleba, V. Klimyuk and S. Marillonnet, "Magnifection-A New Platform for Expressing Recombinant Vaccines in Plants," Vaccine, Vol. 23, No. 17-18, 2005, pp. 2042-2048. http://dx.doi.org/10.1016/j.vaccine.2005.01.006

[26] E. Stoger, J. K. Ma, R. Fischer and P. Christou, "Sowing the Seeds of Success: Pharmaceutical Proteins from Plants," Current Opinion in Biotechnology, Vol. 16, No. 2, 2005, pp. 167-173. http://dx.doi.org/10.1016/j.copbio.2005.01.005

[27] D. Lee and E. Natesan, "Evaluating Genetic Containment Strategies for Transgenic Plants,” Trends in Biotechnology, Vol. 24, No. 3, 2006, pp. 109-114. http://dx.doi.org/10.1016/j.tibtech.2006.01.006 